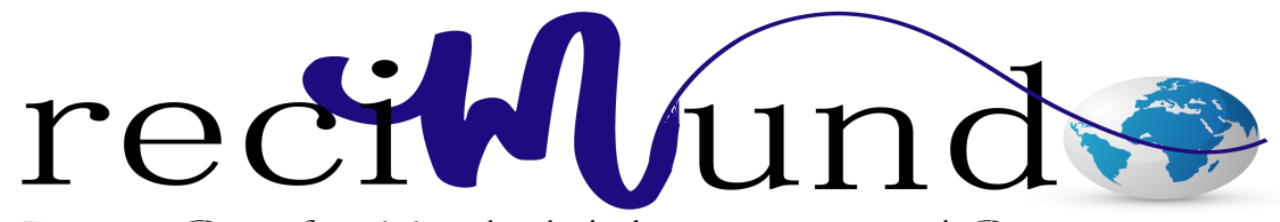

Revista Cientifica Mundo de la Investigación y el Conocimiento

Dixon Dimas Del Valle Pisco ${ }^{\text {a }}$ Narda Aguilera Molina ${ }^{\text {b; }}$ Miguel Eduardo Ganchozo Rivera ${ }^{c}$

Estudio de casos clínicos y terapéuticos de la osteonecrosis de los maxilares como consecuencia de la administración de bifosfonatos

Revista Científica Mundo de la Investigación y el Conocimiento. Vol. 2 núm., especial, mayo, ISSN: 2588-073X, 2018, pp. 177-196

DOI: 10.26820/recimundo/2.esp.2018.177-196

Editorial Saberes del Conocimiento

Recibido: 05/12/2017

Aceptado: 15/02/2018
a. oddixondp@gmail.com
b. Universidad de Guayaquil; narda.aguilera@ug.edu.ec
c. miguel_ganchozo@hotmail.com 


\section{Estudio de casos clínicos y terapéuticos de la osteonecrosis de los maxilares como consecuencia de la administración de bifosfonatos}

Vol. 2, núm. Esp., (2018)

Dixon Dimas Del Valle Pisco; Narda Aguilera Molina; Miguel Eduardo Ganchozo Rivera

\section{RESUMEN}

En el presente trabajo se realizó una investigación sobre la osteonecrosis de los maxilares en pacientes a los que se les administra bifosfonatos. Esta patología es la exposición crónica de hueso necrótico que se presenta en tres estadios clínicos y se encuentra relacionado con estos fármacos cuando son indicados por larga duración en tratamientos para cáncer, osteoporosis, enfermedad de Paget, entre otras. El objetivo se encamino en analizar casos clínicos y tratamientos indicados para la osteonecrosis de los maxilares como consecuencia de la administración de bifosfonatos. Se reportó por primera vez en el año 2003 hasta la actualidad y debido a su morbilidad; los odontólogos y cirujanos maxilofaciales deben saber de esta patología, para así diagnosticar, prevenir y realizar el respectivo tratamiento. Para la investigación de tipo bibliográfico, descriptivo y no experimental se tomaron casos clínicos publicados por distintos autores y se analizó la incidencia, edad, sexo, tipo y modo de administración, efectos colaterales, tiempo de permanecía en el tratamiento de bifosfonatos, localización, sintomatología y elección de tratamiento que cada autor realizo a cada paciente; como resultado se obtuvo que hay una mayor incidencia en mujeres que se les administran estos fármacos para la osteoporosis, la aparición de la Osteonecrosis maxilar se da después de un tratamiento odontológico invasivo, en su mayoría extracciones de piezas dentarias, que acudieron a consulta cuando presentaban un estadio II de evolución y en su mayoría el tratamiento de elección fue la administración de antibióticos combinada con la disminución de la frecuencia de uso del fármaco. Se recomienda una conducta conservadora y menos invasiva posible, con utilización de antisépticos locales, antibióticos y desbridamiento óseo superficial en exposiciones amplias; reservándose la resección quirúrgica y debe ser evaluado constantemente junto al médico tratante.

Palabras Clave: Bifosfonatos, Osteonecrosis Maxilar, Casos clínicos, prevención, pacientes oncológicos. 


\title{
Estudio de casos clínicos y terapéuticos de la osteonecrosis de los maxilares como consecuencia de la administración de bifosfonatos
}

Vol. 2, núm. Esp., (2018)

Dixon Dimas Del Valle Pisco; Narda Aguilera Molina; Miguel Eduardo Ganchozo Rivera

\begin{abstract}
In this paper an investigation of osteonecrosis of the jaw in patients who are administered bisphosphonates was conducted. This condition is chronic exposure of necrotic bone that occurs in three clinical stages and is related to these drugs when indicated by full-length treatments for cancer, osteoporosis, Paget's disease, among others. The aim is headed in analyzing clinical cases and treatments indicated for osteonecrosis of the jaw as a result of the administration of bisphosphonates. It was first reported in 2003 to the present and due to its morbidity; dentists and maxillofacial surgeons should be aware of this disease, in order to diagnose, prevent and make the respective treatment. For bibliographic research, descriptive and non-experimental clinical cases reported by different authors and incidence, age, sex, type and mode of administration, side effects, time remaining in the treatment of bisphosphonates, location, symptoms analyzed were taken and choice of treatment each author each patient; as a result it was found that there is a higher incidence in women who are given these drugs for osteoporosis, the appearance of the jaw Osteonecrosis occurs after an invasive dental treatment, mostly teeth removal, who consulted when presented stage II of evolution and mostly was the treatment of choice administering antibiotics combined with decreasing frequency of use of the drug. A conservative approach and less invasive as possible, using local antiseptics, antibiotics and debridement bone surface in large exposures recommended; reserving surgical resection and it should be constantly evaluated by the treating physician.
\end{abstract}

Keywords: Bisphosphonates, osteonecrosis jaw, clinical cases, prevention, cancer patients. 


\section{Estudio de casos clínicos y terapéuticos de la osteonecrosis de los maxilares como consecuencia de la administración de bifosfonatos}

Vol. 2, núm. Esp., (2018)

Dixon Dimas Del Valle Pisco; Narda Aguilera Molina; Miguel Eduardo Ganchozo Rivera

\section{Introducción.}

Los bifosfonatos son potentes inhibidores de los osteoclastos ejerciendo una fuerte interacción con estas células, produciendo una marcada disminución de la reabsorción ósea. (1) . Nos recuerda que los bifosfonatos se utilizan habitualmente en los pacientes con cáncer avanzado, metástasis óseas e hipercalcemia maligna, entre otras, además, individuos con ciertas patologías que afectan el sistema óseo, el proceso de aposición y reabsorción ósea también puede estar alterada. También nos da a conocer que los relatos sobre el uso de los bifosfonatos y consecuente necrosis de la mandíbula han sido descritos en la literatura. La necrosis es caracterizada por la muerte del hueso que resulta como una consecuencia natural de una variada gama de factores sistémicos y locales que comprometen la vascularización ósea ${ }^{1-10}$.

La osteonecrosis es una enfermedad relacionada con la administración de bifosfonatos, afecta a las estructuras dentoalveolares limitando o inhibiendo la capacidad de reparación del hueso, en estos casos una intervención quirúrgica por sí sola no es recomendable ya que debido a la administración del bifosfonato existe una disfunción regenerativa del tejido óseo, con lo cual tan solo extraer quirúrgicamente el tejido necrótico no sería del todo eficaz, ya que de igual manera quedaría debilitado el hueso afectado, algunos pacientes responden con antibioterapia, siendo útil las penicilinas asociadas con inhibidores de la penicilinas, conjuntamente con irrigaciones locales con clorexidina al $0,12 \%$ y desbridamiento menores de secuestros óseos; estudios recientes han asociado factores de crecimiento como plasma rico en plaqueta con la finalidad de estimular la angiogénesis y reparación del tejido óseo local, en este procedimiento 


\section{Estudio de casos clínicos y terapéuticos de la osteonecrosis de los maxilares como consecuencia de la administración de bifosfonatos}

Vol. 2, núm. Esp., (2018)

Dixon Dimas Del Valle Pisco; Narda Aguilera Molina; Miguel Eduardo Ganchozo Rivera todo el hueso alveolar es removido, restando solamente el componente basal, colocando el plasma rico en plaqueta, finalizando con la sutura la mucosa ${ }^{11-20}$.

Como explicación de la frecuente localización en los maxilares, se ha resaltado el estrés fisiológico a que son sometidas dichas áreas, únicas en exponer hueso al medio externo. Este estrés aumenta por procesos patológicos dentales y periodontales: abscesos, iatrogenia (tratamiento endodóntico, extracciones dentales y otros), que exigen aumentar la velocidad de recambio o remodelación ósea, la que se encuentra bloqueada por dichas drogas, a esto se agrega el efecto antiangiogénico de los bifosfonatos, que provocan disminución del factor de crecimiento de endotelios, posiblemente explicando la mayor frecuencia de compromiso de la mandíbula (63-80\%), hueso de menor irrigación en relación al maxilar superior (14-38\%). La mayoría de los casos de osteonecrosis se presentan posterior a una extracción dental (78\%), infección u otro proceso y sólo una minoría (14\%) aparece espontáneamente. Sólo 5,5\% se presentan en ambos maxilares ${ }^{21-25}$.

Explica que los bifosfonatos tienen una vida media de años, aun no se ha establecido una relación causa efecto de estas drogas sobre la Osteonecrosis maxilar, sin embargo la incidencia en tiempos anteriores en relación al uso de bifosfonatos era muy baja, siendo que hoy día alcanza $10 \%$ o más en las patologías mencionadas tratadas con zoledronato y algo menos con otros ${ }^{25-30}$.

El objetivo de un estudio de osteonecrosis asociada a la administración de bifosfonatos, se sustenta por el aumento de casos de pacientes con cáncer y osteoporosis a los cuales se les administra bifosfonatos, siendo estos muy buenos para el tratamiento de estas enfermedades, estos pacientes en su gran mayoría son adultos que pasan los 40 años, cuando estos son 


\section{Estudio de casos clínicos y terapéuticos de la osteonecrosis de los maxilares como consecuencia de la administración de bifosfonatos}

Vol. 2, núm. Esp., (2018)

Dixon Dimas Del Valle Pisco; Narda Aguilera Molina; Miguel Eduardo Ganchozo Rivera

sometidos a un tratamiento odontológico invasivo por lo general extracciones se ven altamente expuestos a la aparición de osteonecrosis, motivo por el cual el odontólogo debe estar preparado para afrontar el tratamiento a este tipo de pacientes, de ese modo prevenir la aparición de complicaciones odontológicas.

\section{Metodología.}

Diseño de la Investigación

\section{No Experimental}

Esta investigación se realiza de manera bibliográfica para conocer la causa por la cual se desarrolla la osteonecrosis maxilar asociada a la administración de bifosfonatos, y de igual manera conocer el tratamiento utilizado por los diversos autores en los pacientes.

El diseño de esta investigación es no experimental, ya que se realiza mediante el análisis de los casos clínicos de pacientes que padecieron osteonecrosis, realizados por diversos autores, se espera encontrar respuestas al problema planteado mediante el uso de los datos recopilados en la investigación.

\section{Métodos Teóricos}

Inductivo - Deductivo

Por ser un tema de gran relevancia en nuestra profesión donde existen varios estudios en diferentes artículos científicos me ha permitido realiza esta investigación con la recopilación de 


\section{Estudio de casos clínicos y terapéuticos de la osteonecrosis de los maxilares como consecuencia de la administración de bifosfonatos}

Vol. 2, núm. Esp., (2018)

Dixon Dimas Del Valle Pisco; Narda Aguilera Molina; Miguel Eduardo Ganchozo Rivera diferentes criterios y resultados que nos va a servir como base de conocimiento y que se logra llegar a mis propios resultados, conclusiones y recomendaciones mediante la deducción

\section{Analítico - Sintético}

Esta investigación es analítica ya que recolectará información de caso de pacientes que presentaban osteonecrosis de los maxilares por bifosfonatos para analizar cómo se presentan las manifestaciones clínicas y los tratamientos que existe para cada estadio de esta patología por varios autores. Y es sintético porque luego de es recolección de datos se procederá a realizar una tabulación de resultados y a mencionar las conclusiones de nuestro trabajo.

\section{Técnicas}

Nuestra investigación se basara al análisis bibliográfico en la cual utilizamos artículos científicos relacionados al tema, y también de la biblioteca para la recolección de información. Se buscara los casos clínicos relevantes de pacientes atendidos por presentar Osteonecrosis mandibular asociada al uso de bifosfonatos. Se analizara los datos obtenidos. Y se expondrán los resultados.

\section{Herramientas Estadísticas}

Se utilizara información bibliográfica de los diferentes casos clínicos actuales referente al tema 


\section{Estudio de casos clínicos y terapéuticos de la osteonecrosis de los maxilares como consecuencia de la administración de bifosfonatos}

Vol. 2, núm. Esp., (2018)

Dixon Dimas Del Valle Pisco; Narda Aguilera Molina; Miguel Eduardo Ganchozo Rivera

Tipos de Investigación

Según la naturaleza de los objetivos en cuanto al nivel de conocimiento que se desea alcanzar, el tipo de investigación que se aplico fue:

Documental.- Porque se realizara a través de la revisión de documentos científicos publicados que se enfocan en el estudio de los bifosfonatos, su uso, ventajas y desventajas, de igual modo su influencia en la aparición de la osteonecrosis maxilar junto a la revisión de casos clínicos presentados por varios autores.

Descriptiva.- Esta investigación está enfocada en describir los aspectos terapéuticos y tóxicos de los bifosfonatos principalmente en los maxilares, de igual manera se realizara la investigación descriptiva de la osteonecrosis maxilar, sus signos y síntomas clínicos, su incidencia y las pautas terapéuticas que han empleado los diversos autores en sus casos.

Exploratoria.- Debido a que los objetivos de la investigación son principalmente exponer los métodos diagnósticos y terapéuticos que utilizaron los diversos autores para tratar a los pacientes que presentaron Osteonecrosis asociada al uso de bifosfonatos, mediante la exploración analítica de los casos clínicos expuestos

Explicativa.- Se encarga de buscar el porqué de los hechos mediante el establecimiento de relaciones causa - efecto. En este sentido, se estudiara como se da la aparición de la Osteonecrosis maxilar, las causas que llevan a su aparición y de igual manera las formas de prevenir la aparición de la misma, así mismo las pautas de tratamiento. 


\section{Estudio de casos clínicos y terapéuticos de la osteonecrosis de los maxilares como consecuencia de la administración de bifosfonatos}

Vol. 2, núm. Esp., (2018)

Dixon Dimas Del Valle Pisco; Narda Aguilera Molina; Miguel Eduardo Ganchozo Rivera

\section{Población y muestra}

Esta investigación es de tipo bibliográfico descriptivo por esta razón se utilizaran casos clínicos publicados por diversos autores.

\section{Fases metodológicas}

- Podríamos decir, que este proceso tiene tres fases claramente delimitadas:

- Fase conceptual

- Fase metodológica

- Fase empírica

La fase conceptual de la investigación es aquella que va desde la concepción del problema de investigación a la concreción de los objetivos del estudio que pretendemos llevar a cabo. Esta es una fase de fundamentación del problema en el que el investigador descubre la pertinencia y la viabilidad de su investigación, o por el contrario, encuentra el resultado de su pregunta en el análisis de lo que otros han investigado.

La formulación de la pregunta de investigación es: ¿Cuáles son las manifestaciones clínicas y tratamientos indicados para la osteonecrosis de los maxilares como consecuencia de la administración de bifosfonatos?

Revisión bibliográfica de lo que otros autores han investigado sobre nuestro tema de investigación, que nos ayude a justificar y concretar nuestro problema de investigación. 


\section{Estudio de casos clínicos y terapéuticos de la osteonecrosis de los maxilares como consecuencia de la administración de bifosfonatos}

Vol. 2, núm. Esp., (2018)

Dixon Dimas Del Valle Pisco; Narda Aguilera Molina; Miguel Eduardo Ganchozo Rivera

Descripción del marco de referencia de nuestro estudio: Para darnos cuenta de esta patología la osteonecrosis de los maxilares que a nivel de odontología no se la conoce.

Relación de los objetivos e hipótesis de la investigación: Analizar casos clínicos y tratamientos indicados para la osteonecrosis de los maxilares como consecuencia de la administración de bifosfonatos.

La fase metodológica es una fase de diseño, en la que la idea toma forma. En esta fase dibujamos el "traje" que le hemos confeccionado a nuestro estudio a partir de nuestra idea original. Sin una conceptualización adecuada del problema de investigación en la fase anterior, resulta muy difícil poder concretar las partes que forman parte de nuestro diseño:

Elección del diseño de investigación: El diseño de esta investigación es no experimental, descriptivo, exploratorio, bibliográfico, ya que mediante el análisis de los casos clínicos realizados por diversos autores se espera encontrar respuestas al problema planteado.

Definición de los sujetos del estudio: Esta investigación es de tipo bibliográfico descriptivo por esta razón no cuenta con análisis de universo y muestra, ya que se utilizaran casos clínicos publicados por diversos autores.

Descripción de las variables de la investigación: El objeto de estudio es Administración de bifosfonatos y el campo de acción es Osteonecrosis de los maxilares. Estas dos variables tiene una gran relación a nivel odontológico en la cual debemos conocer sus manifestaciones clínicas y tratamientos adecuados para cada estadio. 


\section{Estudio de casos clínicos y terapéuticos de la osteonecrosis de los maxilares como consecuencia de la administración de bifosfonatos}

Vol. 2, núm. Esp., (2018)

Dixon Dimas Del Valle Pisco; Narda Aguilera Molina; Miguel Eduardo Ganchozo Rivera

Elección de las herramientas de recogida y análisis de los datos: Para la investigación se tomaron casos clínicos publicados de distintos autores y se analizó la incidencia, edad, sexo, tipo y modo de administración, efectos colaterales, el tiempo de permanecía en el tratamiento de bifosfonatos, localización, sintomatología y la elección de tratamiento que cada autor realizo a cada paciente

La última fase, la fase empírica es, sin duda, la que nos resulta más atractiva, Recogida de datos: En esta etapa recogeremos los datos de forma sistemática utilizando las herramientas que hemos diseña do previamente. Análisis de los datos: Los datos se analizan en función de la finalidad del estudio, según se pretenda explorar o describir fenómenos o verificar relaciones entre variables.

Interpretación de los resultados:

Un análisis meramente descriptivo de los datos obtenidos puede resultar poco interesante, tanto para el investigador, como para los interesados en conocer los resultados de un determinado estudio. Poner en relación los datos obtenidos con el contexto en el que tienen lugar y analizarlo a la luz de trabajos anteriores enriquece, sin duda, el estudio llevado a cabo.

Difusión de los resultados: Una investigación que no llega al resto de la comunidad de personas y profesionales implicados en el objeto de la misma tiene escasa utilidad, aparte de la satisfacción personal de haberla llevado a cabo. Si pensamos que la investigación mejora la práctica clínica comunicar los resultados de la investigación resulta un deber ineludible para cualquier investigador. 


\section{Estudio de casos clínicos y terapéuticos de la osteonecrosis de los maxilares como consecuencia de la administración de bifosfonatos}

Vol. 2, núm. Esp., (2018)

Dixon Dimas Del Valle Pisco; Narda Aguilera Molina; Miguel Eduardo Ganchozo Rivera

\section{Resultados.}

Después de haber realizado la recopilación de 15 artículos científicos actualizados referente a nuestro tema de diversas páginas del sitio web, se realizó su respectivo análisis de los casos clínicos y síntesis de los resultados que arrojaron en cada uno de sus artículos, para proceder a realizar una tabulación con los datos obtenidos y dar los siguientes resultados:

\section{Grafico.- Osteonecrosis de los maxilares por administración de bifosfonatos}

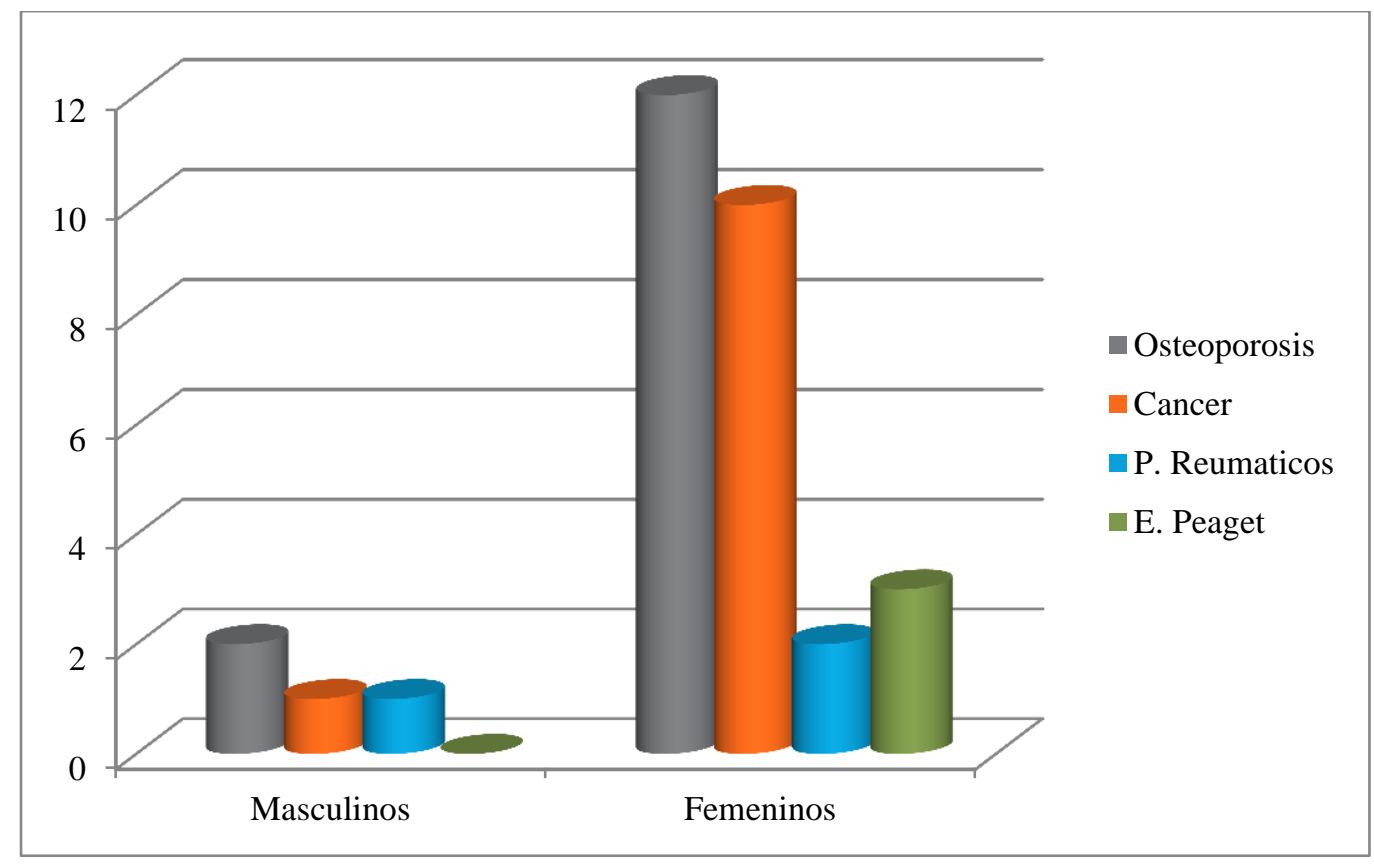

Análisis: En los casos investigados se encontró que a los pacientes que se les han administrado bifosfonatos en un periodo superior a 24 meses, se encontró que en la mayoría de los casos lo pacientes son de sexo femeninos y la razón principal de la administración de los bifosfonatos fue porque presentaban la enfermedad de osteoporosis. 


\section{Estudio de casos clínicos y terapéuticos de la osteonecrosis de los maxilares como consecuencia de la administración de bifosfonatos}

Vol. 2, núm. Esp., (2018)

Dixon Dimas Del Valle Pisco; Narda Aguilera Molina; Miguel Eduardo Ganchozo Rivera

\section{Grafico $N^{\circ}$ 2.- Bifosfonatos y su porcentaje de prescripción}



Análisis: Observamos los porcentajes de los bifosfonatos mas recetados y administrados. Entre ellos tenemos los bifosfonatos con mayor administración en los casos clínicos revisados fue el Pamidronat con un 35\%, el 3G Acido Zoledrónico con 30\%, en menor medida Ibandronato, Etidronato, Clodronato con el $15 \%$ y el $10 \%$ respectivamente. 
Estudio de casos clínicos y terapéuticos de la osteonecrosis de los maxilares como consecuencia de la administración de bifosfonatos

Vol. 2, núm. Esp., (2018)

Dixon Dimas Del Valle Pisco; Narda Aguilera Molina; Miguel Eduardo Ganchozo Rivera

\section{Grafico $N^{\circ}$ 3.- Causas de la osteonecrosis}

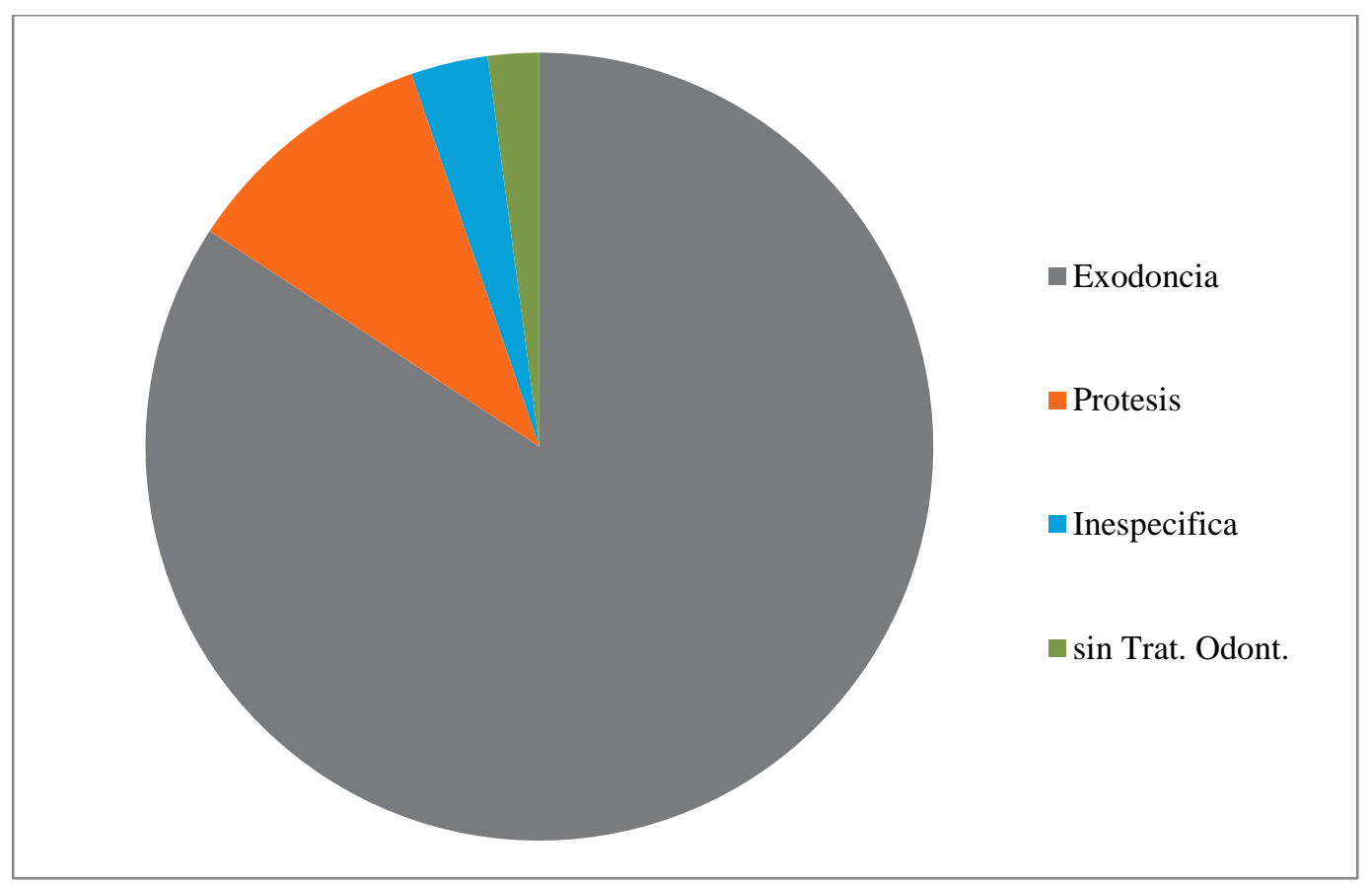

Análisis: En los casos revisados de los artículos llegamos que la aparición de la Osteonecrosis se presentó luego de la realización de una intervención dental quirúrgica, principalmente exodoncias.

Otros factores incluyen el escaso espesor de la mucosa maxilar-mandibular y la frecuente "agresión" a la que se ve sometido el hueso en forma de procedimientos quirúrgicos u odontológicos invasivos. 


\section{Estudio de casos clínicos y terapéuticos de la osteonecrosis de los maxilares como consecuencia de la administración de bifosfonatos}

Vol. 2, núm. Esp., (2018)

Dixon Dimas Del Valle Pisco; Narda Aguilera Molina; Miguel Eduardo Ganchozo Rivera

\section{Grafico $N^{\circ}$ 4.- Estadios evolutivos de la osteonecrosis}



Análisis: En nuestra investigación encontramos que los pacientes en los casos clínicos presentaron una evolución de estadio II en un $80 \%$, el $15 \%$ presento estadio III y un $5 \%$ presento estadio I.

Estos pacientes en estadio II presenta en la cavidad oral una zona de tumefacción y enrojecimiento submandibular en relación con una zona de osteonecrosis en boca. 
Estudio de casos clínicos y terapéuticos de la osteonecrosis de los maxilares como consecuencia de la administración de bifosfonatos

Vol. 2, núm. Esp., (2018)

Dixon Dimas Del Valle Pisco; Narda Aguilera Molina; Miguel Eduardo Ganchozo Rivera

\section{Grafico $N^{\circ}$ 5.- Tratamiento de la osteonecrosis}

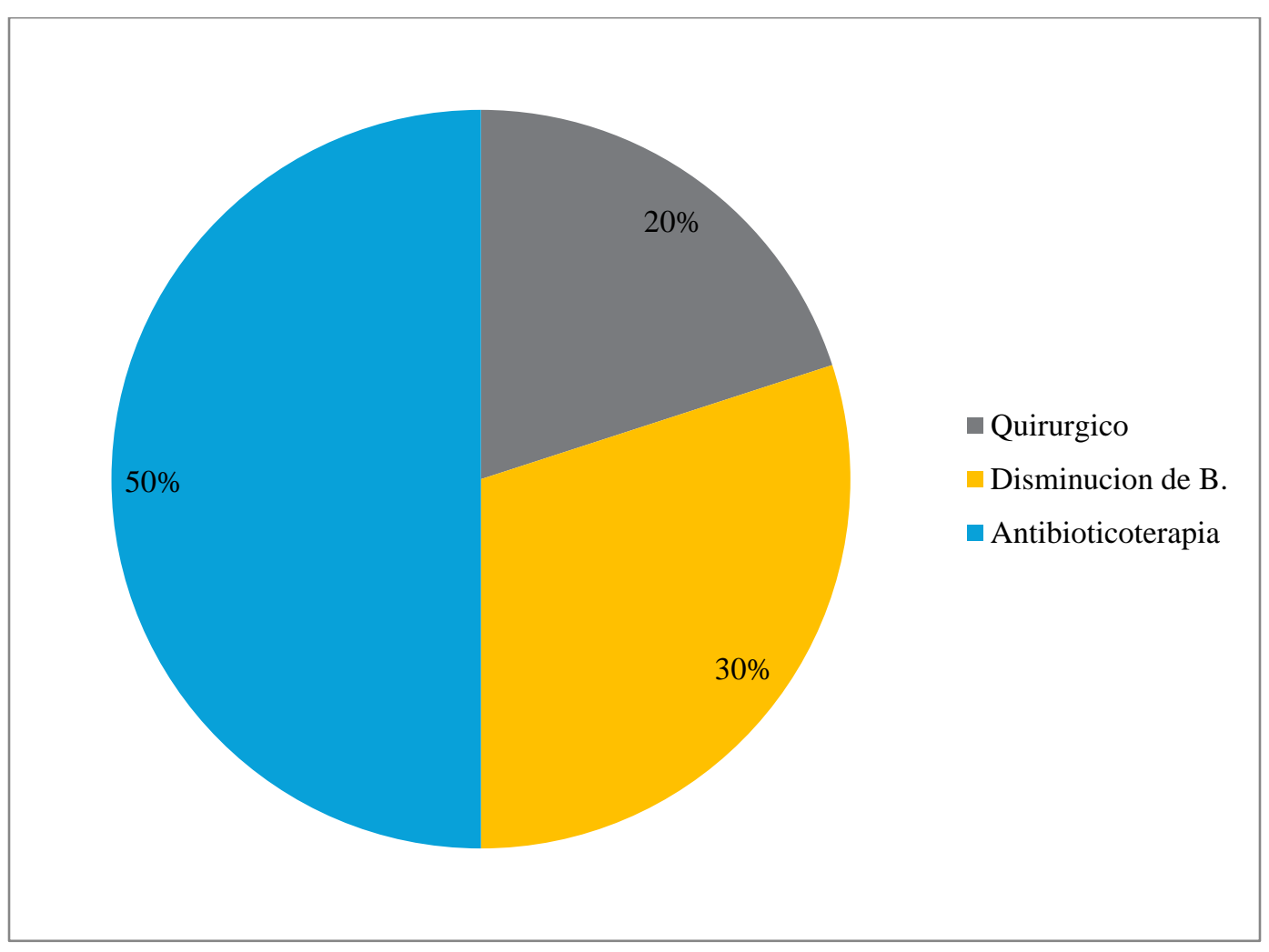

Análisis: El tratamiento más utilizado por distintos autores fue la disminución de la dosis de bifosfonatos, junto a la administración de antibióticos, de preferencia se evitó las intervenciones quirúrgicas.

Recomienda cuidados locales con clorhexidina gel o solución, se agrega el uso de AINES y antibioterapia para evitar la infección, que es una de las causas de progresión de la lesión. Amoxicilina/clavulánico 875/125 mg cada 8 horas, por un período de 15 días. 


\section{Estudio de casos clínicos y terapéuticos de la osteonecrosis de los maxilares como consecuencia de la administración de bifosfonatos}

Vol. 2, núm. Esp., (2018)

Dixon Dimas Del Valle Pisco; Narda Aguilera Molina; Miguel Eduardo Ganchozo Rivera

\section{Conclusiones.}

Luego de haber hecho una revisión bibliográfica de los casos clínicos de una serie de pacientes con el diagnóstico de osteonecrosis de los maxilares, establezco las siguientes con conclusiones:

- Para diagnosticar la posible aparición de la Osteonecrosis de los maxilares por administración de bifosfonatos, es necesario estudiar y relacionar la edad del paciente, la enfermedad de base, el tipo de bifosfonato, vía de administración, dosis, tiempo promedio de la medicación, así como todos aquellos factores locales y sistémicos que se pertenecen con la aparición de la entidad

- La mayor incidencia se encuentra en mujeres, principalmente aparece en pacientes tratados con bifosfonatos intravenosos que padecen osteoporosis y que mantienen el tratamiento durante un periodo mayor a 24 meses.

- Principalmente la aparición de la Osteonecrosis se produce después de la exodoncia de una pieza dental.

- Los pacientes en su mayoría acuden a consulta cuando cruzan por el estadio II de evolución de la Osteonecrosis.

- El tratamiento recurrente escogido por los diversos autores es el tratamiento conservador, no quirúrgico, antibiótico, acompañado de una disminución de la frecuencia de administración del bifosfonato, aunque si se realizó terapéutica quirúrgica, esta fue exclusiva para los pacientes que presentaban Osteonecrosis en estadio III 


\section{Estudio de casos clínicos y terapéuticos de la osteonecrosis de los maxilares como consecuencia de la administración de bifosfonatos}

Vol. 2, núm. Esp., (2018)

Dixon Dimas Del Valle Pisco; Narda Aguilera Molina; Miguel Eduardo Ganchozo Rivera

- $\quad$ Los bifosfonatos se demuestran mayormente en pacientes de edad avanzada

- Antes de comenzar una administración de bifosfonatos se debe realizar una interconsulta con el odontólogo y por ende seguir el protocolo recomendado.

- La prevención es el mejor tratamiento para esta enfermedad, con una buena atención médico-odontólogo

\section{Bibliografía.}

1. Jaimes M. Bifosfonatos y Osteonecrosis de los Maxilares. Consideraciones Sobre su Tratamiento. Int. J. Morphol.. 2008; 26(3).

2. Jiménez SY. Los bifosfonatos, nueva causa de osteonecrosis maxilar por fármacos: situación actual.. Med. Oral Patol. Oral Cir. Bucal. 2010.

3. Hardman JG, Limbrid LE, Molinoff PB, Ruddon RW, Gilman AG. Goodman \& Gilman's. Las bases farmacológicas de la terapéutica. 19th ed. Mexico: McGraw-Hill-Interamericana; 2008.

4. Medina A. Alternativas de tratamiento para la osteonecrosis de los maxilares asociada a bisfosfonatos. Avances en Odontoestomatología. 2010; 26(3).

5. Capote C. CONSIDERACIONES EN EL TRATAMIENTO ODONTOLÓGICO DE PACIENTES EN TERAPIA CON BIFOSFONATOS. Acta Odontologica Venezolana. 2010; 49(4).

6. Lezcano B. Osteonecrosis de los maxilares inducida por bifosfonatos: prevención y actitud terapéutica. Revista Española de Cirugía Oral y Maxilofacial. 2007; 29(5).

7. Martínez MÁ. Osteonecrosis. ¿Qué hay de nuevo? Reumatol Clinica. 2009.

8. Sánchez A. Osteonecrosis de la Mandíbula. Revista Española de Cirugía Oral y Maxilofacial. 2013; 48(3).

9. Henríquez S. Osteonecrosis de los maxilares. Osteoporos Metab Miner. 2009; 1(1).

10. Riancho J. Bifosfonatos en la prevención y tratamiento de la osteoporosis. 8th ed. Madrid: FHOEMO; 2010. 


\section{Estudio de casos clínicos y terapéuticos de la osteonecrosis de los maxilares como consecuencia de la administración de bifosfonatos}

Vol. 2, núm. Esp., (2018)

Dixon Dimas Del Valle Pisco; Narda Aguilera Molina; Miguel Eduardo Ganchozo Rivera

11. Sanchez Dj. Diccionario de especialidades farmacéuticas PLM Mexico: PLM ; 2012.

12. Bagán J. Recomendaciones para la prevención de la osteonecrosis de los maxilares en pacientes con cáncer tratados con bifosfonatos. Med Oral Patol Oral Cir Bucal. 2008.

13. Vitte C. Resorcion de osteoclastos inducida por bifosfonatos. Endocrinology. 2009.

14. Gutierrez J. OSTEONECROSIS MANDIBULAR RELACIONADAS CON EL USO DE BIFOSFONATOS. SECIB. 2008.

15. Tovar J. APLICACIÓN DE FIBRINA RICA EN PLAQUETAS EN LA CICATRIZACIÓN Y REGENERACION POST-EXODONCIAS DENTARIAS EN PACIENTES CON RIESGO Y CON OSTEONECROSIS MAXILAR INDUCIDA POR BIFOSFONATOS. Caracas: UNIVERSIDAD CENTRAL DE VENEZUELA.; 2011.

16. Renée S. Tratamiento de la osteoporosis. Tomo II. Montevideo: Sociedad uruguaya de reumatología; 2009.

17. Barrientos FJ. Osteonecrosis de los maxilares inducida por bifosfonatos: prevención y actitud terapéutica. Revista Española de Cirugía Oral y Maxilofacial. 2007; 29(5).

18. García F. OSTEONECROSIS MANDIBULAR RELACIONADAS CON EL USO DE BIFOSFONATOS.CASOS CLÍNICOS. Secib. 2011.

19. Bocanegra S. Osteonecrosis maxilar secundaria al uso de bisfosfonatos por vía oral. Exposición de tres casos clínicos relacionados con alendronato. Revista médica de Chile. 2009.

20. Jorge G. Osteonecrosis de los maxilares inducida por bifosfonatos; lo que el odontólogo debe saber hoy: pautas y protocolos. Odontoestomatología. 2013; 15(21).

21. Pastor D. Osteonecrosis maxilar y bisfosfonatos.. Medicina Oral, Patología Oral y Cirugía Bucal. 2006.

22. Otero J. Osteonecrosis de los maxilares asociada al uso de bifosfonatos: revisión de ocho casos. Revista Española de Cirugía Oral y Maxilofacial. 2011.

23. Dmedicina edr. http://www.dmedicina.com. [Online].; 2010 [cited 2015 febrero 27. Available from: http://www.dmedicina.com/enfermedades/musculos-y-huesos/osteoporosis.

24. medlineplus edr. http://www.nlm.nih.gov. [Online].; 2014 [cited 2015 febrero 27. Available from: http://www.nlm.nih.gov/medlineplus/spanish/ency/article/001289.htm. 
Estudio de casos clínicos y terapéuticos de la osteonecrosis de los maxilares como consecuencia de la administración de bifosfonatos

Vol. 2, núm. Esp., (2018)

Dixon Dimas Del Valle Pisco; Narda Aguilera Molina; Miguel Eduardo Ganchozo Rivera

25. JA C. Osteonecrosis avascular inducida por bifosfonatos. Universidad central de Venezuela, Facultad de odontologia. 2008.

26. Garcia J. Efectividad del legrado óseo q uirúrgico como tratamiento de la osteoquimionecrosis mandibular México; 2010.

27. Casal C. Osteonecrosis de maxilares relacionada con el uso de bifosfonatos. ELSEIVER. 2012; 10(1).

28. Jaramillo R. Incidencia de osteonecrosis de los maxilares inducido por bifosfonatos post tratamiento implantológico Madrid: UNIVERSIDAD COMPLUTENSE DE MADRID ; 2013.

29. Blanco A. INFLUENCIA DE LOS BIFOSFONATOS SOBRE EL MOVIMIENTO DENTARIO ORTODÓNCICO Caracas: Universidad Central de Venezuela; 2010.

30. Cardona F. Osteonecrosis de los maxilares por bisfosfonatos. Actualización y puesta al día. Anales del Sistema Sanitario de Navarra. 2009. 